\title{
Association between body fat parameters and arterial stiffness
}

\author{
Hack-Lyoung Kim ${ }^{1}$, Dong-Won Ahn², Su Hwan Kim², Dong Seok Lee², Soon Ho Yoon ${ }^{3}$, \\ Joo-Hee Zo1 ${ }^{1}$, Myung-A. Kim ${ }^{1} \&$ Ji Bong Jeong ${ }^{2 \bowtie}$
}

The influence of body fat on arterial stiffness remains controversial. This study was performed to investigate the associations between four different types of body fat parameters and brachial-ankle pulse wave velocity (baPWV). A total of 3758 subjects (mean age, $53.4 \pm 8.8$ years; females, 36.3\%) who underwent health check-up were retrospectively analyzed. Anthropometric parameters including body mass index (BMI), waist circumference (WC) and waist-hip ratio (WHR) were assessed, and visceral fat area (VFA) was calculated by bioelectrical impedance analysis. In simple linear correlation analyses, baPWV was associated with WC, WHR and VFA $(P<0.001$ for each), but not with BMI $(P=0.175)$. In multivariable analyses, $B M I$ and WC were not associated with baPWV $(P>0.05$ for each). Even after controlling for potential confounders, higher baPWV was significantly associated not only with higher WHR [for $>0.90$ in men and $>0.85$ in women: odds ratio (OR), $1.23 ; 95 \%$ confidence interval (CI), 1.06-1.42; $P=0.005$; for the highest tertile compared to the lowest tertile: $O R, 1.38 ; 95 \% \mathrm{Cl}$, $1.15-1.66 ; P<0.001$ ], but also with higher VFA (for $\geq 100 \mathrm{~cm}^{2}$ : OR, 1.39; $95 \% \mathrm{Cl}, 1.20-1.60 ; P<0.001$; for the highest tertile compared to the lowest tertile: $O R, 1.77 ; 95 \% \mathrm{Cl}, 1.48-2.12 ; P<0.001)$. Our study showed that baPWV was correlated with WHR and VFA, but not with BMI and WC. This implies that arterial stiffness may be more strongly associated with abdominal obesity than overall obesity.

\author{
Abbreviations \\ BMI Body mass index \\ baPWV Brachial-ankle pulse wave velocity \\ CI Confidence interval \\ IRB Institutional Review Board \\ OR Odds ratio \\ WC Waist circumference \\ WHR Waist-hip ratio \\ VFA Visceral fat area
}

Arterial wall gradually stiffened due to aging and prolonged exposure to various stressful conditions such as high blood pressure, hyperglycemia, dyslipidemia, smoking and inflammation ${ }^{1,2}$. Although there are various methods to measure arterial stiffness, pulse wave velocity is the most widely used because of its non-invasiveness, simplicity and rich clinical data ${ }^{3}$. Of clinical significance, the information on arterial stiffness has predicted future cardiovascular events in a variety of patients as well as the general population independent of traditional risk factors ${ }^{4-6}$. Therefore, it is important to find factors related to arterial stiffness because it can be applied to cardiovascular prevention and treatment strategies.

As the number of obese people around the world is steadily increasing and the morbidities related to obesity have become a big problem in human society ${ }^{7,8}$. Anthropometric parameters and other indicators related to obesity and body fat in our bodies are receiving increasing attention. Given that the association between obesity and cardiovascular disease is well established ${ }^{9,10}$, vascular dysfunction has been suggested as one of the factors linking these two pathological states ${ }^{11}$. However, the relationship between arterial stiffness and adiposity is still inconsistent ${ }^{12-15}$. It is also known that cardiovascular risk varies depending on the location of body fat in our body: visceral fat is more strongly associated with cardiovascular risk than subcutaneous fat ${ }^{16-18}$. Considering

\footnotetext{
${ }^{1}$ Division of Cardiology, Department of Internal Medicine, Boramae Medical Center, Seoul National University College of Medicine, Seoul, South Korea. ${ }^{2}$ Division of Gastroenterology and Hepatology, Department of Internal Medicine, Boramae Medical Center, Seoul National University College of Medicine, 20 Borame-ro 5-gil, Dongjak-gu, Seoul 07061, South Korea. ${ }^{3}$ Department of Radiology, Seoul National University College of Medicine, Seoul National University Hospital, Seoul, South Korea. ${ }^{\circledR}$ email: jibjeong@snu.ac.kr
} 


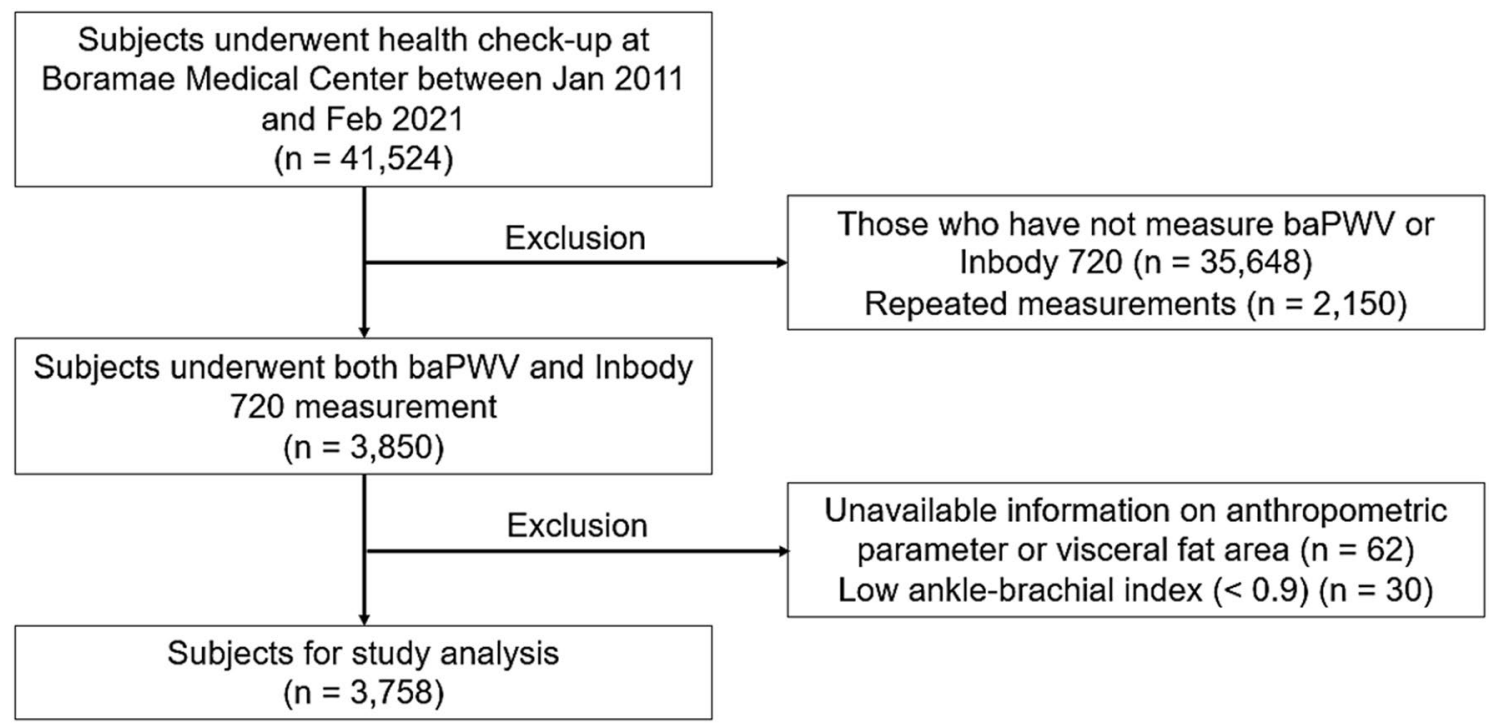

Figure 1. Flow chart of enrollment of study subjects. $b a P W V$ brachial-ankle pulse wave velocity.

this, it would be valuable to examine the relationships between measures of arterial stiffness and obesity according to the location of body fat.

In this study, we investigated the associations of brachial-ankle PWV (baPWV) with several parameters of body fat including body mass index (BMI), waist circumference (WC), waist-hip ratio (WHR) and visceral fat area (VFA). Our hypothesis was that indicators of abdominal fat including WC, WHR and VFA, were more significantly associated with baPWV than that an indicator of overall obesity, BMI.

\section{Methods}

Study population. This single center and the retrospective study was performed at a general hospital in a big city (Seoul, South Korea). Between January 2011 and February 2021, both baPWV and Inbody 720 measurements were made in 3850 of 41,524 subjects who underwent voluntary health check-up at the health care center of Boramae Medical Center (Seoul, South Korea). After exclusion of 92 subjects with unavailable information on anthropometric parameter of VFA $(n=62)$, and low ankle-brachial index $(<0.9)(n=30), 3758$ subjects were finally analyzed in this study. The study flow of subject enrollment is demonstrated in Fig. 1 . This study conforms to the ethical guidelines of the Declaration of Helsinki, and the study protocol was approved by the Institutional Review Board (IRB) of Boramae Medical Center (Seoul, Korea) (IRB number, 30-2021-95). Obtaining informed consent was waived by the IRB of Boramae Medical Center (Seoul, Korea) due to retrospective study design and routine nature of data collected.

Clinical data. Blood pressure was measured on the right upper arm using an oscillometric device by a trained nurse. Hypertension was defined as systolic blood pressure $\geq 140 \mathrm{mmHg}$, diastolic blood pressure $\geq 90 \mathrm{mmHg}$, or the use of anti-hypertensive medications. Diabetes mellitus was defined as fasting plasma glucose $\geq 126 \mathrm{mg} / \mathrm{dL}$, glycated hemoglobin $\geq 6.5 \%$, or anti-diabetic medications. Dyslipidemia was defined as triglyceride $\geq 150 \mathrm{mg} /$ $\mathrm{dL}$, high-density lipoprotein $<40 \mathrm{mg} / \mathrm{dL}$ in men and $<50 \mathrm{mg} / \mathrm{dL}$ in women, or the use of anti-dyslipidemic medications. Information on cigarette smoking, alcohol drinking, and previous history of coronary artery disease and stroke was obtained based on the questionnaires. After overnight fasting for about $12 \mathrm{~h}$, blood levels of the following parameters were obtained using commercially available chemistry analyzer (Modular DP and Cobas 8000, Roche. Diagnostics, Mannheim, Germany; G11vr, Tosoh Bioscience, Inc., Tokyo, Japan): glucose, glycated hemoglobin, total cholesterol, low-density lipoprotein cholesterol, high-density lipoprotein cholesterol, triglyceride, aspartate transaminase, alanine aminotransferase, gamma-glutamyl transferase and C-reactive protein.

Anthropometric data. At the day of health check-up height and body weight were measured. BMI was calculated as body weight divided by height squared $\left(\mathrm{kg} / \mathrm{m}^{2}\right)$. Normal weight, overweight and obesity were defined as $\mathrm{BMI}<23 \mathrm{~kg} / \mathrm{m}^{2}, 23 \sim 24.9 \mathrm{~kg} / \mathrm{m}^{2}$, and $\geq 25 \mathrm{~kg} / \mathrm{m}^{2}$, respectively ${ }^{19}$. WC was measured by a trained nurse while exhaling in a standing position just above the hip bone with a tape measure. During the measurement, the tape was hold flat against body, not too tight and take a reading. Abdominal obesity was defined as WC $\geq 90 \mathrm{~cm}$ in men and $\geq 85 \mathrm{~cm}$ in women ${ }^{19}$. Hip circumference was measured around the widest portion of the buttocks, with the tape parallel to the floor. WHR was calculated as WC/hip circumference. Abdominal obesity was defined as WHR $>0.90 \mathrm{~cm}$ in men and $>0.85 \mathrm{~cm}$ in women ${ }^{20}$.

VFA. VFA was automatically calculated by bioelectrical impedance analysis using Inbody 720 (Biospace Co., Seoul, South Korea ${ }^{21}$. It has been reported that VFA measurements using the Inbody 720 well correlated with the results of computed tomography $(\mathrm{CT})^{22}$. In our health screening cohort, there was 2441 subjects underwent 
CT examination. Pearson's bivariate correlation analysis showed a significant and strong correlation between VFA measured by INbody 720 and by CT $(r=0.718, P<0.001)$ (Supplementary Figure S1). A subject with VFA $\geq 100 \mathrm{~cm}^{2}$ was considered to have visceral obesity ${ }^{23}$.

baPWV. The baPWV was automatically generated using a VP-1000 analyzer (Collin Co., Komaki, Japan) ${ }^{24,25}$. Measurement of baPWV was performed in an isolated room with constant temperature and humidity in the morning. All subjects were fasting, taking no cardiovascular medications, and resting in a supine position for at least five minutes prior to measurement. Blood pressure cuffs were wrapped around the upper arms and both ankles, and pressure wave forms of the brachial and tibial arteries were recorded with plethysmographic and oscillometric pressure sensors. Time differences between the points where the pulse waveform starts to rise in the systolic phase in the brachial and tibial arteries were measured. The distance between the two points was automatically calculated from the subject's height, and baPWV was calculated by dividing the distance between the two points by the time difference. The average value of right and left baPWV was used in our study analysis. Measurement of baPWV was performed by a single experienced expert. The coefficient of variation in baPWV measurement for intra-observer variability was $5.1 \%$ in our laboratory ${ }^{26}$.

Statistical analysis. Continuous variables are expressed as mean \pm standard deviation, and categorical variables are expressed as $\mathrm{n}(\%)$. Study subjects were stratified into two groups according to the median value of baPWV: subjects with higher baPWV $(\geq 1372 \mathrm{~cm} / \mathrm{s})$ and those with lower baPWV $(<1372 \mathrm{~cm} / \mathrm{s})$. Continuous variables were compared using Student's $t$-test, and categorical variables were compared using the $\chi^{2}$ test between the two groups. The simple linear correlation between two continuous variables was analyzed using Pearson's bivariate correlation analysis, and demonstrated using scatter plot. Binary multiple logistic regression analysis was performed to find independent associations of anthropometric measures and VFA with baPWV. The following clinical covariates were adjusted in this multivariable analysis: age, sex, hypertension, diabetes mellitus, dyslipidemia, cigarette smoking and alcohol drinking. Multiple linear regression analysis was also performed to show independent associations between body fat parameters and baPWV after controlling for age and sex. $P$ value of $<0.05$ was considered statistically significant. All statistical analyses were performed using SPSS 22.0 (IBM Corp., Armonk, NY, USA).

\section{Results}

The clinical characteristics of 3,758 total study subjects and comparisons of clinical characteristics between subjects with higher $(\geq 1372 \mathrm{~cm} / \mathrm{s})$ and lower $(<1372 \mathrm{~cm} / \mathrm{s})$ baPWV are demonstrated in Table 1 . In total subjects, mean age was $53.4 \pm 8.8$ years and male predominant $(63.7 \%)$. The prevalence of hypertension, diabetes mellitus and dyslipidemia, and previous history of coronary artery disease and stroke were $21.9 \%, 8.6 \%, 15.5 \%, 1.3 \%$, and $0.4 \%$, respectively. The results of main laboratory tests were within normal limits. Subjects with higher baPWV $(\geq 1372 \mathrm{~cm} / \mathrm{s})$ were older and had higher blood pressure than those with lower baPWV $(<1372 \mathrm{~cm} / \mathrm{s})$. Cardiovascular risk factors including hypertension, diabetes mellitus, dyslipidemia and previous coronary artery disease were more prevalent in subjects with higher baPWV than those with lower baPWV. The results of laboratory tests were unfavorably presented in subjects with higher baPWV than those with lower baPWV. The parameters of body fat are shown in Table 2. Although there was a somewhat difference according to the parameters, about one third (27.5-41.1\%) were obese. All 4 body fat parameters including BMI, WC, WHR and VFA were significantly higher in subjects with higher baPWV than those with lower baPWV. In simple linear correlation analyses, baPWV was associated with WC, WHR and VFA $(P<0.001$ for each), but not with BMI $(P=0.175)$ (Table 3). These associations are demonstrated as scatter plots in Fig. 2. In multivariable binary logistic regression analyses, BMI and WC were not associated with baPWV $(P>0.05$ for each). Even after controlling for potential confounders, higher baPWV was significantly associated with not only with higher WHR (for $>0.90$ in men and $>0.85$ in women: odds ratio [OR], $1.23 ; 95 \%$ confidence interval $[\mathrm{CI}], 1.06-1.42 ; P=0.005$; for the highest tertile compared to the lowest tertile: OR, 1.38; 95\% CI, 1.15-1.66; $P<0.001$ ), but also with higher VFA (for $\geq 100$ $\mathrm{cm}^{2}$ : OR, 1.39; 95\% CI, 1.20-1.60; $P<0.001$; for the highest tertile compared to the lowest tertile: OR, 1.77; $95 \%$ CI, 1.48-2.12; $P<0.001$ ) were significantly associated with higher baPWV (Table 4 ).

In multiple linear regression analyses, baPWV was associated with WC $(\beta=0.085 ; P<0.001)$, WHR $(\beta=0.081$; $P<0.001)$ and VFA $(\beta=0.116 ; P<0.001)$ but not with BMI $(\mathrm{P}=0.412)$ even after controlling for age and sex (Supplementary Table S1). In age-specific analysis (Supplementary Table S2), WHR and VFA were more strongly associated with baPWV in young age group ( $<55$ years) than in those older age group ( $\geq 55$ years). Both BMI and WC were not associated with baPWV in both age groups.

\section{Discussion}

Our study showed that baPWV correlated more strongly with WHR and VFA than with BMI and WC. BMI was not correlated with baPWV even in simple correlation analysis. Although WC was positively correlated with baPWV in univariable analysis, its significance disappeared after controlling for potential confounders in multivariable logistic regression analysis. The correlations of baPWV with WHR and VFA remained even after controlling for important clinical covariates. The correlation between baPWV and VFA was strongest. Given that the degree of abdominal obesity is better expressed by WC, WHR and VFA than by BMI, these results suggest that abdominal obesity is more strongly associated with arterial stiffness than overall obesity.

Previous studies on the association between body fat parameters and arterial stiffness. There are several studies that looked at the association between body fat parameters and arterial stiffness ${ }^{12-15,27-31}$. We summarized the results of these studies (Supplementary Table S3). Their results are inconsistent and do not 


\begin{tabular}{|c|c|c|c|c|}
\hline Characteristic & Total subjects $(\mathrm{n}=3758)$ & $\begin{array}{l}\text { Subjects with baPWV } \geq 1372 \mathrm{~cm} / \mathrm{s} \\
(\mathrm{n}=1885)\end{array}$ & $\begin{array}{l}\text { Subjects with baPWV }<1372 \mathrm{~cm} / \mathrm{s} \\
(\mathrm{n}=1873)\end{array}$ & $P$ \\
\hline Age, years & $53.4 \pm 8.8$ & $56.6 \pm 8.1$ & $50.2 \pm 8.3$ & $<0.001$ \\
\hline Female sex & $1,363(36.3)$ & $608(32.3)$ & $755(40.3)$ & $<0.001$ \\
\hline Systolic BP, mmHg & $123 \pm 14$ & $130 \pm 13$ & $115 \pm 10$ & $<0.001$ \\
\hline Diastolic BP, mmHg & $76.8 \pm 10.4$ & $81.8 \pm 9.5$ & $71.9 \pm 8.8$ & $<0.001$ \\
\hline \multicolumn{5}{|l|}{ Cardiovascular risk factors } \\
\hline Hypertension & $822(21.9)$ & $589(31.2)$ & $233(12.4)$ & $<0.001$ \\
\hline Diabetes mellitus & $324(8.6)$ & $253(13.4)$ & $71(3.8)$ & $<0.001$ \\
\hline Dyslipidemia & $582(15.5)$ & $378(20.1)$ & $204(10.9)$ & $<0.001$ \\
\hline Cigarette smoking & $480(12.8)$ & $229(12.1)$ & $251(13.4)$ & 0.250 \\
\hline Alcohol drinking & $702(18.7)$ & $366(19.4)$ & $336(17.9)$ & 0.245 \\
\hline Previous CAD & $50(1.3)$ & $38(2.0)$ & $12(0.6)$ & $<0.001$ \\
\hline Previous stroke & $14(0.4)$ & $10(0.5)$ & $4(0.2)$ & 0.111 \\
\hline \multicolumn{5}{|l|}{ Laboratory findings } \\
\hline Fasting glucose, mg/dL & $97.2 \pm 21.1$ & $101.7 \pm 24.7$ & $92.6 \pm 15.3$ & $<0.001$ \\
\hline Glycated hemoglobin, \% & $5.76 \pm 0.76$ & $5.9 \pm 0.9$ & $5.5 \pm 0.4$ & $<0.001$ \\
\hline Total cholesterol, mg/dL & $195 \pm 36$ & $195 \pm 38$ & $195 \pm 33$ & 0.965 \\
\hline LDL cholesterol, mg/dL & $119 \pm 33$ & $119 \pm 36$ & $119 \pm 31$ & 0.998 \\
\hline HDL cholesterol, mg/dL & $54.3 \pm 13.9$ & $53.1 \pm 13.3$ & $55.6 \pm 14.4$ & $<0.001$ \\
\hline Triglyceride, mg/dL & $113 \pm 71$ & $120 \pm 73$ & $106 \pm 67$ & $<0.001$ \\
\hline AST, IU/L & $27.8 \pm 12.7$ & $29.1 \pm 13.5$ & $26.4 \pm 11.7$ & $<0.001$ \\
\hline ALT, IU/L & $27.9 \pm 18.5$ & $29.5 \pm 13.5$ & $26.2 \pm 17.4$ & $<0.001$ \\
\hline GGT, IU/L & $33.4 \pm 44.8$ & $38.2 \pm 56.5$ & $28.7 \pm 27.8$ & $<0.001$ \\
\hline C-reactive protein, $\mathrm{mg} / \mathrm{L}$ & $1.45 \pm 4.67$ & $0.16 \pm 0.59$ & $0.12 \pm 0.28$ & 0.010 \\
\hline
\end{tabular}

Table 1. Clinical characteristics of study subjects according to baPWV. Numbers are expressed as mean \pm standard deviation or $\mathrm{n}(\%) . b a P W V$ brachial-ankle pulse wave velocity, $B P$ blood pressure, $C A D$ coronary artery disease, $L D L$ low-density lipoprotein, $H D L$ high-density lipoprotein, $A S T$ aspartate transaminase, $A L T$ alanine aminotransferase, GGT gamma-glutamyl transferase.

\begin{tabular}{|c|c|c|c|c|}
\hline Parameter & Total subjects $(n=3758)$ & Subjects with baPWV $\geq 1372 \mathrm{~cm} / \mathrm{s}(\mathrm{n}=1885)$ & Subjects with baPWV $<1372 \mathrm{~cm} / \mathrm{s}(\mathrm{n}=1873)$ & $P$ \\
\hline Body mass index, $\mathrm{kg} / \mathrm{m}^{2}$ & $24.0 \pm 3.1$ & $24.1 \pm 2.9$ & $23.9 \pm 3.2$ & 0.048 \\
\hline Body mass index $\geq 25 \mathrm{~kg} / \mathrm{m}^{2}$ & $1369(36.4)$ & $710(37.7)$ & $659(35.2)$ & 0.114 \\
\hline Waist circumference, $\mathrm{cm}$ & $83.1 \pm 9.3$ & $84.0 \pm 8.9$ & $82.1 \pm 9.5$ & $<0.001$ \\
\hline $\begin{array}{l}\text { Waist circumference, } m e n \geq 90 \mathrm{~cm} \text {, } \\
\text { women } \geq 85 \mathrm{~cm}\end{array}$ & $1035(27.5)$ & $567(30.1)$ & $468(25.0)$ & $<0.001$ \\
\hline Waist-hip ratio & $0.87 \pm 0.04$ & $0.88 \pm 0.04$ & $0.87 \pm 0.04$ & $<0.001$ \\
\hline Waist-hip ratio, men $>0.90$, women $>0.85$ & $1545(41.1)$ & $836(44.4)$ & $709(37.9)$ & $<0.001$ \\
\hline Visceral fat area, $\mathrm{cm}^{2}$ & $94.2 \pm 32.0$ & $99.2 \pm 31.6$ & $89.1 \pm 31.5$ & $<0.001$ \\
\hline Visceral fat area $\geq 100 \mathrm{~cm}^{2}$ & $1466(39.0)$ & $858(45.5)$ & $608(32.5)$ & $<0.001$ \\
\hline
\end{tabular}

Table 2. Body fat parameters according to baPWV. baPWV brachial-ankle pulse wave velocity.

\begin{tabular}{|l|l|r|}
\hline Parameter & $\boldsymbol{r}$ & $\boldsymbol{P}$ \\
\hline Body mass index & 0.022 & 0.175 \\
\hline Waist circumference & 0.123 & $<0.001$ \\
\hline Waist-hip ratio & 0.115 & $<0.001$ \\
\hline Visceral fat area & 0.170 & $<0.001$ \\
\hline
\end{tabular}

Table 3. Simple linear correlations showing the associations between body fat parameters and baPWV. $b a P W V$ brachial-ankle pulse wave velocity. 

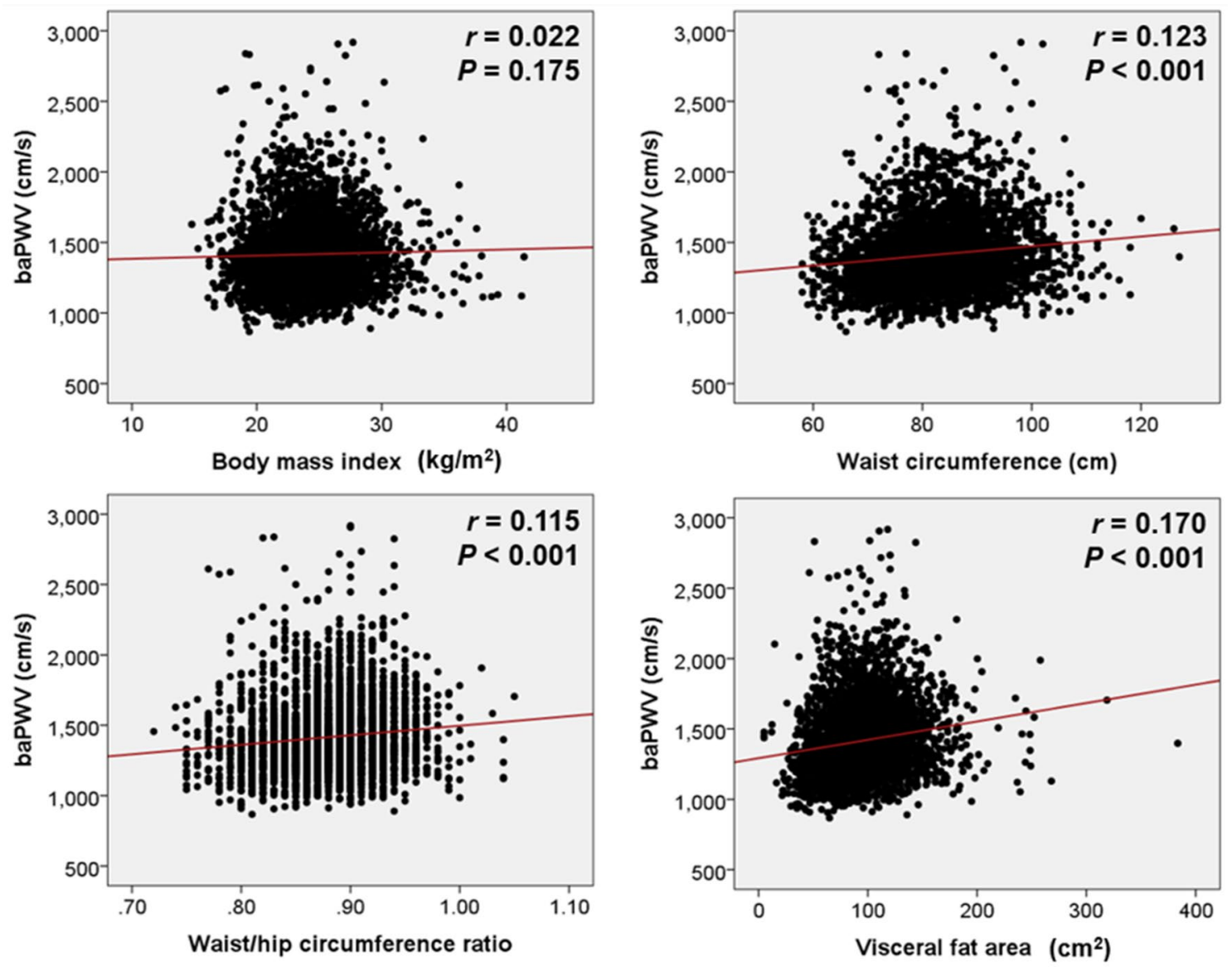

Figure 2. Scatter plots showing correlations of baPWV with four body fat parameters. $b a P W V$ brachial-ankle pulse wave velocity.

\begin{tabular}{|l|l|r|}
\hline Parameter & OR $(\mathbf{9 5} \% \mathbf{C I})$ & \multicolumn{1}{|l|}{} \\
\hline Body mass index $\geq 25 \mathrm{~kg} / \mathrm{m}^{2}$ & $0.86(0.74-1.00)$ & 0.051 \\
\hline Body mass index $<23 \mathrm{~kg} / \mathrm{m}^{2}$ & 1 & \\
\hline Body mass index, $23-24.9 \mathrm{~kg} / \mathrm{m}^{2}$ & $1.11(0.93-1.33)$ & 0.209 \\
\hline Body mass index $\geq 25 \mathrm{~kg} / \mathrm{m}^{2}$ & $0.91(0.77-1.07)$ & 0.271 \\
\hline Body mass index, the lowest tertile & 1 & \\
\hline Body mass index, middle tertile & $1.09(0.92-1.29)$ & 0.276 \\
\hline Body mass index, the highest tertile & $0.86(0.72-1.03)$ & 0.113 \\
\hline Waist circumference, men $\geq 90 \mathrm{~cm}$, women $\geq 85 \mathrm{~cm}$ & $1.02(0.87-1.19)$ & 0.756 \\
\hline Waist circumference, the lowest tertile & 1 & \\
\hline Waist circumference, middle tertile & $1.26(1.06-1.50)$ & 0.007 \\
\hline Waist circumference, the highest tertile & $1.10(0.91-1.32)$ & 0.307 \\
\hline Waist-hip ratio, men $>0.90$, women $>0.85$ & $1.23(1.06-1.42)$ & 0.005 \\
\hline Waist-hip ratio, the lowest tertile & 1 & \\
\hline Waist-hip ratio, middle tertile & $1.43(1.21-1.68)$ & $<0.001$ \\
\hline Waist-hip ratio, the highest tertile & $1.38(1.15-1.66)$ & $<0.001$ \\
\hline Visceral fat area $\geq 100$ cm ${ }^{2}$ & $1.39(1.20-1.60)$ & $<0.001$ \\
\hline Visceral fat area, the lowest tertile & 1 & \\
\hline Visceral fat area, middle tertile & $1.52(1.28-1.80)$ & $<0.001$ \\
\hline Visceral fat area, the highest tertile & $1.77(1.48-2.12)$ & $<0.001$ \\
\hline
\end{tabular}

Table 4. Multiple binary logistic regression analyses showing independent associations of body fat parameters with higher baPWV $(\geq 1372 \mathrm{~cm} / \mathrm{s})$. Following clinical covariates are controlled: age, sex, hypertension, diabetes mellitus, dyslipidemia, cigarette smoking and alcohol drinking. baPWV brachial-ankle pulse wave velocity, $O R$ odds ratio, CI confidence interval. 
conclusively establish an association of arterial stiffness with body fat parameters. In addition, body fat parameters used in most of these studies are anthropometric indices ${ }^{12-14,27,28,31}$. The use of a more objective indicator, VFA, has been used in only a few studies ${ }^{15,29,30}$, but the number of patients analyzed in these is relatively small (50-344 subjects) ${ }^{15,29,30}$. On the other hand, in our study, we used VFA as well as anthropometric indices, and the number of study subjects was very large with 3758 subjects. In addition, although it is relatively well-known that the location of fat in our body has different effects on the cardiovascular system, there are few studies that analyzed the association between measures of arterial stiffness and obesity according to the location of body fat. We used both overall (BMI) and visceral obesity (WC, WHR and VFA) indices for analysis, and suggested that the visceral obesity index is more correlated with arterial stiffness than overall obesity index.

Although not focused on the association between obesity indices and arterial stiffness according to the location of body fat, a few studies have addressed this issue ${ }^{27-30}$. In a study of 146 middle-aged adults, both carotid-femoral pulse wave velocity (cfPWV) and baPWV had stronger associations with WC and VFA than with $\mathrm{BMI}^{27}$. A study that examined 10,197 Chinese subjects who underwent health check-up showed that baPWV was more strongly correlated with WHR than BMI and $\mathrm{WC}^{28}$. In a study of 344 patients who underwent kidney transplantation, WHR and VFA were significantly associated with baPWV and cfPWV in univariable analysis; however, the association disappeared in multivariable analysi ${ }^{29}$. Sex difference was shown in some studies. Nordstrand and colleagues have shown that increasing BMI, WC, WHR and VFA were independently associated with higher cfPWV in women but not in men among 133 morbidly obese patients ${ }^{30}$. Similar findings were observed in another study of 2647 healthy individuals demonstrating that baPWV correlated with BMI and WC only in women but not in men $^{31}$. All of these studies, including ours indicate that abdominal obesity is more strongly associated with arterial stiffness than overall obesity.

Pathophysiology. Abdominal obesity is more harmful to cardiovascular system than overall obesity ${ }^{17}$. Visceral fat secretes a variety of cytokines, leading to chronic inflammatory conditions, endothelial cell dysfunction and insulin resistance ${ }^{17,32,33}$. These unfavorable factors may also contribute to vascular pathology such as increased arterial stiffness ${ }^{27,34}$. In particular, increased circulating leptin level elevates blood pressure and sympathetic tone, leading to arterial stiffening ${ }^{35}$. Also, shared traditional cardiovascular risk factors such as high blood pressure, hyperglycemia, dyslipidemia and cigarette smoking can explain the association between abdominal obesity and arterial stiffening ${ }^{36}$. It is inferred that increased arterial stiffness may be at least partially responsible for poor cardiovascular prognosis in patients with abdominal obesity. Also, the opposite hypothesis is possible: increased cardiovascular risk in patients with increased arterial stiffness may be attributable in part to abdominal obesity.

Clinical implications. For physicians treating obesity, improving poor cardiovascular prognosis in obese patients is critical. In this regard, it is important to use indicators of obesity that are more strongly correlated with cardiovascular risk. Although BMI, as an overall obesity indicator, is the simplest and most widely used in clinical practice, it does not reflect muscle mass or fat distribution. Our findings, along with previous studies, showed that arterial stiffness, a good predictor of cardiovascular events ${ }^{5,6}$, was more frequently associated with indicators of abdominal obesity than BMI. Considering that the measurement of VFA requires specific equipment and technology, it is recommended to use WHR rather than of BMI as an indicator of obesity in clinical practice. WC is another indicator of abdominal obesity; however, it may underestimate risk in smaller people. Indeed, in our findings, WC correlated more strongly with baPWV than BMI, but not with WHR or VFA.

Several studies have reported improvement in arterial stiffness with weight loss ${ }^{37}$ and decreased VFA ${ }^{38}$. Other studies have found improvement in various metabolic factors and endothelial cell function in patients with abdominal obesity following therapeutic lifestyle intervention ${ }^{39,40}$. This indicates that it can improve arterial stiffness by controlling abdominal obesity. Based on the data so far, perhaps the best way to improve both arterial stiffness and abdominal obesity is aerobic exercise $\mathrm{e}^{41,42}$. However, further research is needed to determine whether arterial stiffness improved by control of abdominal obesity leads to reduced cardiovascular risk.

We showed that the association between visceral adiposity and baPWV was more pronounced in younger subjects than older ones (Supplementary Table S2). Although underlying pathophysiology to explain this agerelated difference is not clear, it is likely that other risk factors influencing arterial stiffness may play a stronger role with age. From a clinical point of view, this may mean that more aggressive management of abdominal obesity in young subjects is needed to reduce cardiovascular risk. However, more research results are needed to support our findings.

Study limitations. This study has several limitations. First, as this is a cross-sectional study, the causal relationship between obesity and arterial stiffness could not be established. Second, data on concomitant medications were not available in our study. There was a possibility that some vasoactive medications had an influence on arterial stiffness. Lastly, the study population was restricted to Korean adults, so direct application of our results to other ethnic groups is difficult.

\section{Conclusions}

Our study showed that baPWV was significantly correlated with WHR and VFA, but not with BMI. This implies that arterial stiffness may be more strongly associated with abdominal obesity than with overall obesity. Further studies with a larger sample size are needed to confirm our findings.

\section{Data availability}

All data generated or analyzed during this study are included in this article. 
Received: 11 August 2021; Accepted: 23 September 2021

Published online: 15 October 2021

\section{References}

1. Lee, H. Y. \& Oh, B. H. Aging and arterial stiffness. Circ. J. 74, 2257-2262. https://doi.org/10.1253/circj.cj-10-0910 (2010).

2. Cavalcante, J. L., Lima, J. A., Redheuil, A. \& Al-Mallah, M. H. Aortic stiffness: Current understanding and future directions. J. Am. Coll. Cardiol. 57, 1511-1522. https://doi.org/10.1016/j.jacc.2010.12.017 (2011).

3. Laurent, S. et al. Expert consensus document on arterial stiffness: Methodological issues and clinical applications. Eur. Heart J. 27, 2588-2605. https://doi.org/10.1093/eurheartj/ehl254 (2006).

4. Chirinos, J. A., Segers, P., Hughes, T. \& Townsend, R. Large-artery stiffness in health and disease: JACC state-of-the-art review. J. Am. Coll. Cardiol. 74, 1237-1263 (2019).

5. Vlachopoulos, C., Aznaouridis, K. \& Stefanadis, C. Prediction of cardiovascular events and all-cause mortality with arterial stiffness: A systematic review and meta-analysis. J. Am. Coll. Cardiol. 55, 1318-1327. https://doi.org/10.1016/j.jacc.2009.10.061 (2010).

6. Ohkuma, T. et al. Brachial-ankle pulse wave velocity and the risk prediction of cardiovascular disease: An individual participant data meta-analysis. Hypertension 69, 1045-1052. https://doi.org/10.1161/hypertensionaha.117.09097 (2017).

7. Afshin, A. et al. Health effects of overweight and obesity in 195 countries over 25 years. N. Engl. J. Med. 377, 13-27 (2017).

8. Ng, M. et al. Global, regional, and national prevalence of overweight and obesity in children and adults during 1980-2013: A systematic analysis for the Global Burden of Disease Study 2013. Lancet 384, 766-781 (2014).

9. Whitlock, G. et al. Body-mass index and cause-specific mortality in 900000 adults: Collaborative analyses of 57 prospective studies. Lancet 373, 1083-1096 (2009).

10. Jousilahti, P., Tuomilehto, J., Vartiainen, E., Pekkanen, J. \& Puska, P. Body weight, cardiovascular risk factors, and coronary mortality: 15-year follow-up of middle-aged men and women in eastern Finland. Circulation 93, 1372-1379. https://doi.org/10.1161/01. cir.93.7.1372 (1996).

11. Bastien, M., Poirier, P., Lemieux, I. \& Després, J. P. Overview of epidemiology and contribution of obesity to cardiovascular disease. Prog. Cardiovasc. Dis. 56, 369-381. https://doi.org/10.1016/j.pcad.2013.10.016 (2014).

12. Wildman, R. P., Mackey, R. H., Bostom, A., Thompson, T. \& Sutton-Tyrrell, K. Measures of obesity are associated with vascular stiffness in young and older adults. Hypertension 42, 468-473. https://doi.org/10.1161/01.hyp.0000090360.78539.cd (2003).

13. Rodrigues, S. L. et al. Body mass index is not independently associated with increased aortic stiffness in a Brazilian population. Am. J. Hypertens. 25, 1064-1069. https://doi.org/10.1038/ajh.2012.91 (2012).

14. Tang, B. et al. Relationship between body mass index and arterial stiffness in a health assessment Chinese population. Medicine (Baltimore) 99, e18793 (2020).

15. Rider, O. J. et al. The effect of obesity and weight loss on aortic pulse wave velocity as assessed by magnetic resonance imaging. Obesity (Silver Spring) 18, 2311-2316. https://doi.org/10.1038/oby.2010.64 (2010).

16. Czernichow, S., Kengne, A. P., Stamatakis, E., Hamer, M. \& Batty, G. D. Body mass index, waist circumference and waist-hip ratio: which is the better discriminator of cardiovascular disease mortality risk?: Evidence from an individual-participant meta-analysis of 82864 participants from nine cohort studies. Obes. Rev. 12, 680-687 (2011).

17. Després, J. P. Body fat distribution and risk of cardiovascular disease: An update. Circulation 126, 1301-1313. https://doi.org/10. 1161/circulationaha.111.067264 (2012)

18. Yusuf, S. et al. Obesity and the risk of myocardial infarction in 27,000 participants from 52 countries: A case-control study. Lancet 366, 1640-1649. https://doi.org/10.1016/s0140-6736(05)67663-5 (2005).

19. Seo, M. H. et al. 2018 Korean Society for the study of obesity guideline for the management of obesity in Korea. J. Obes. Metab. Syndr. 28, 40-45 (2019).

20. WHO. Waist Circumference and Waist-Hip Ratio. Report of a WHO Expert Consultation Geneva, 8-11 December (2008).

21. Ogawa, H. et al. InBody 720 as a new method of evaluating visceral obesity. Hepatogastroenterology 58, 42-44 (2011).

22. Ahn, D. W. et al. Fatty liver is an independent risk factor for gallbladder polyps. World J. Gastroenterol. 26, 6979-6992 (2020).

23. New criteria for "obesity disease" in Japan. Circ. J. 66, 987-992. https://doi.org/10.1253/circj.66.987 (2002).

24. Yamashina, A. et al. Validity, reproducibility, and clinical significance of noninvasive brachial-ankle pulse wave velocity measurement. Hypertens. Res. 25, 359-364. https://doi.org/10.1291/hypres.25.359 (2002).

25. Kwak, S. et al. Associations of brachial-ankle pulse wave velocity with left ventricular geometry and diastolic function in untreated hypertensive patients. Front. Cardiovasc. Med. 8, 647491 (2021).

26. Lee, H. S. et al. Incremental prognostic value of brachial-ankle pulse wave velocity to single-photon emission computed tomography in patients with suspected coronary artery disease. J. Atheroscler. Thromb. 22, 1040-1050. https://doi.org/10.5551/jat.29918 (2015).

27. Strasser, B. et al. Abdominal obesity is associated with arterial stiffness in middle-aged adults. Nutr. Metab. Cardiovasc. Dis. 25, 495-502. https://doi.org/10.1016/j.numecd.2015.01.002 (2015).

28. Zhang, J. et al. Comparison of the ability to identify arterial stiffness between two new anthropometric indices and classical obesity indices in Chinese adults. Atherosclerosis 263, 263-271. https://doi.org/10.1016/j.atherosclerosis.2017.06.031 (2017).

29. Heleniak, Z. et al. Obesity, fat tissue parameters, and arterial stiffness in renal transplant recipients. Transplant Proc. 52, 2341-2346. https://doi.org/10.1016/j.transproceed.2020.01.118 (2020).

30. Nordstrand, N. et al. The relationship between various measures of obesity and arterial stiffness in morbidly obese patients. $B M C$ Cardiovasc. Disord. 11, 7 (2011).

31. Choi, H. S. et al. Association between new anthropometric parameters and arterial stiffness based on brachial-ankle pulse wave velocity. Diabetes Metab. Syndr. Obes. 12, 1727-1733 (2019).

32. Kahn, S. E., Hull, R. L. \& Utzschneider, K. M. Mechanisms linking obesity to insulin resistance and type 2 diabetes. Nature 444, 840-846. https://doi.org/10.1038/nature05482 (2006).

33. Caballero, A. E. Endothelial dysfunction in obesity and insulin resistance: A road to diabetes and heart disease. Obes. Res. 11, 1278-1289. https://doi.org/10.1038/oby.2003.174 (2003).

34. Fantin, F. et al. Abdominal obesity and subclinical vascular damage in the elderly. J. Hypertens. 28, 333-339. https://doi.org/10. 1097/HJH.0b013e328333d23c (2010).

35. Cheng, K. H. et al. Adipocytokines and proinflammatory mediators from abdominal and epicardial adipose tissue in patients with coronary artery disease. Int. J. Obes. (Lond.) 32, 268-274. https://doi.org/10.1038/sj.ijo.0803726 (2008).

36. Kim, H. L. \& Kim, S. H. Pulse wave velocity in atherosclerosis. Front. Cardiovasc. Med. 6, 41 (2019).

37. Dengo, A. L. et al. Arterial destiffening with weight loss in overweight and obese middle-aged and older adults. Hypertension 55, 855-861 (2010).

38. Yu, H. et al. Decreased visceral fat area correlates with improved arterial stiffness after Roux-en-Y gastric bypass in Chinese obese patients with type 2 diabetes mellitus: A 12-month follow-up. Surg. Obes. Relat. Dis. 12, 550-555. https://doi.org/10.1016/j.soard. 2015.09.003 (2016).

39. Ferré, R. et al. Effects of therapeutic lifestyle changes on peripheral artery tonometry in patients with abdominal obesity. Nutr. Metab. Cardiovasc. Dis. 22, 95-102. https://doi.org/10.1016/j.numecd.2010.04.008 (2012).

40. Voeghtly, L. M. et al. Cardiometabolic risk reduction in an intensive cardiovascular health program. Nutr. Metab. Cardiovasc. Dis. 23, 662-669. https://doi.org/10.1016/j.numecd.2012.01.012 (2013). 
41. Seals, D. R., Desouza, C. A., Donato, A. J. \& Tanaka, H. Habitual exercise and arterial aging. J. Appl. Physiol. 1985(105), 1323-1332 (2008).

42. Montero, D., Roberts, C. K. \& Vinet, A. Effect of aerobic exercise training on arterial stiffness in obese populations : A systematic review and meta-analysis. Sports Med. 44, 833-843. https://doi.org/10.1007/s40279-014-0165-y (2014).

\section{Author contributions}

K.H.L. and J.J.B. designed the study. K.H.L. performed analyses and wrote manuscript. A.D.W., K.S.H., L.D.S., Y.S.H., Z.J.H. and K.M.A. involved in data collection and interpreted the data. J.J.B. revised the manuscript. All authors read and approved the final manuscript.

\section{Competing interests}

The authors declare no competing interests.

\section{Additional information}

Supplementary Information The online version contains supplementary material available at https://doi.org/ 10.1038/s41598-021-00175-Z.

Correspondence and requests for materials should be addressed to J.B.J.

Reprints and permissions information is available at www.nature.com/reprints.

Publisher's note Springer Nature remains neutral with regard to jurisdictional claims in published maps and institutional affiliations.

Open Access This article is licensed under a Creative Commons Attribution 4.0 International License, which permits use, sharing, adaptation, distribution and reproduction in any medium or format, as long as you give appropriate credit to the original author(s) and the source, provide a link to the Creative Commons licence, and indicate if changes were made. The images or other third party material in this article are included in the article's Creative Commons licence, unless indicated otherwise in a credit line to the material. If material is not included in the article's Creative Commons licence and your intended use is not permitted by statutory regulation or exceeds the permitted use, you will need to obtain permission directly from the copyright holder. To view a copy of this licence, visit http://creativecommons.org/licenses/by/4.0/.

(C) The Author(s) 2021 